'A Jamei Khosroshahi, ${ }^{2} \mathrm{~K}$ Sayadpoor, ${ }^{3} \mathrm{~A}$ Kocharian, ${ }^{4} \mathrm{~A}$ Kocharin, ${ }^{5} \mathrm{~A}$ Zeinaloo, ${ }^{4} \mathrm{~A}$ Kiani, ${ }^{4} E$ Malakan, Pediatric Cardiology Group of Tehran University and Urmieh University. 'Urmia University, Urmia; ${ }^{2}$ Pediatric Cardiology, Tehran University of Medical Sciences; ${ }^{3}$ Tabiz University of Medical Science; ${ }^{4}$ Tehran University of Medical Sciences; ${ }^{5}$ Tehran Medical University, Tehran, Iran

Objectives There is not yet a general consensus about the diagnosis of left ventricular noncompaction. The echocardiographer may miss the areas with maximal noncompaction leading to a misdiagnosis. Accordingly, we suggested a new method to measure the percentage of myocardial hypertrabeculation/noncompaction using two-dimensional echocardiography.

Patients In this study, this new method was examined on 3 noncompaction, 26 dilated cardiomyopathy, and 25 normal subjects.

Results The mean percentages of myocardial hypertrabeculation/ noncompaction were $3.59 \pm 2.27$ in control group, $8.86 \pm 5.52$ in dilated cardiomyopathic patients, and $38.1 \pm 30.8$ in noncompaction patients. A value of $16 \%$ could distinguish left ventricular noncompaction from dilated cardiomyopathy with $92 \%$ specificity and $100 \%$ sensitivity and from normal subjects with 100\% specificity and sensitivity. This percentage had a statistically significant association with noncompacted to compacted myocardial thickness ratio $(\mathrm{P}<0.001)$.

Key Conclusions This method showed good correlations with the other echocardiographic and magnetic resonance criteria. However, it is not dependent on finding the area of maximal involvement.

\section{REGIONAL CARDIAC NETWORKS: CAN THEY WORK?}

doi:10.1136/archdischild-2012-302724.0508

${ }^{1} N K$ Puppala, ${ }^{1} \mathrm{R}$ Phatak, ${ }^{1} \mathrm{~K}$ Atasanov, ${ }^{2} \mathrm{~K}$ Parkins, ${ }^{2} \mathrm{~S}$ Ali. ${ }^{1}$ North West \& North Wales Paediatric Transport Service, Central Manchester University Hospitals NHS Foundation Trust, Warrington; ${ }^{2}$ North West \& North UK Paediatric Transport Service, Alder Hey Children's Hospital, Liverpool, Wales

Background Only eleven paediatric intensive care units (PICUs) in UK have cardiac surgical services on-site. Small proportion of patients admitted to non-cardiac surgical PICUs require transport to surgical centres. North West and North Wales Paediatric Transport service (NWTS) provides PIC transport service, including ability to conference call specialists. Alder Hey Children's hospital (AHCH) is the cardiac surgical centre and Royal Manchester Children's hospital (RMCH) only has cardiology on site.

Methods Retrospective review suspected cardiac cases transferred in 12 months:- assessing if time critical surgical patients underwent single transfer to surgical centre- interventions by referring hospital or NWTS.

Results Total 29 patients of suspected cardiac diagnosis.

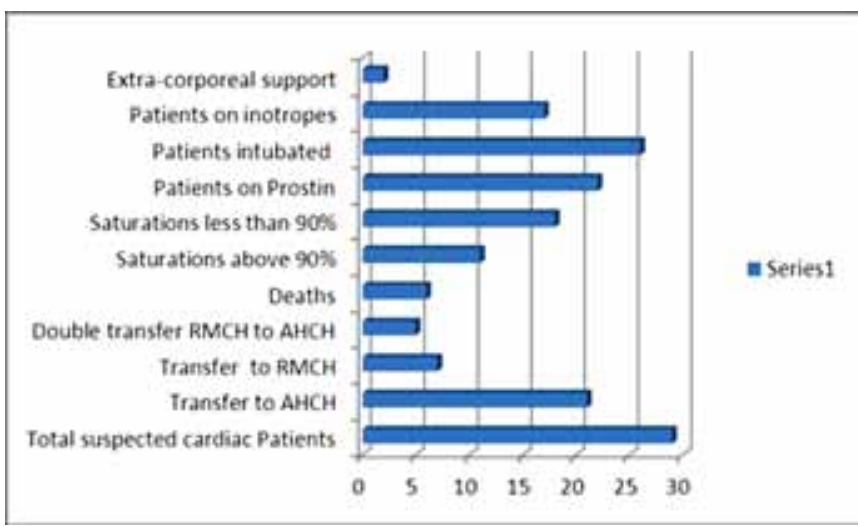

Abstract 508 Graph 1
Abstract 508 Table 1 Interventions

\begin{tabular}{lcc}
\hline INTERVENTIONS & REFERRING HOSPITAL & NWTS \\
\hline Intubation & 22 & 4 \\
Central Venous Access & 6 & 7 \\
Dinoprostone (PGE2) & 20 & 2 \\
Inotropes & 7 & 10 \\
Inhaled Nitric oxide & 0 & 3 \\
\hline
\end{tabular}

Seven patients had time critical cardiac surgical lesions. One patient required a second immediate transfer to surgical centre. Retrospectively, this could have been predicted from clinical picture. $68 \%$ patients had dinoprostone started by referring team following advice from cardiologist or NWTS. Inotropes initiated on advice or by NWTS.

Conclusions Regional cardiac network can work effectively with improved communication particularly in acute scenario. Potential surgical cases may be predicted from clinical picture, especially if not resolving with full medical treatment.

\section{PREVALENCE OF SIDEROPENIC ANEMIA IN CHILDREN WITH CONGENITAL HEART DISEASE LESS THAN ONE YEAR IN COMPARISON WITH HEALTH CHILDREN}

doi:10.1136/archdischild-2012-302724.0509

${ }^{1}$ R Bejiqi, 'R Retkoceri, 'N Zeka, ${ }^{2} \mathrm{H}$ Bejiqi, ${ }^{2} \mathrm{~A}$ Retkoceri, ${ }^{1} \mathrm{~A}$ Gerguri, ${ }^{2} \mathrm{~S}$ Surdulli. ${ }^{\text {P }}$ Pediatric Clinic, University Clinical Center of Kosova, Prishtina; ${ }^{2}$ Main Center of Family Medicine, Prishitna, Kosovo

Introduction Syderopenic anemia is prevalent in all children with congenital heart disease, especially in children with signs of heart failure.

Objectives The purpose of this study is to determine the prevalence of sideropenic anemia in children with CHD and compares these results with data of health children less than one year in Kosova population.

Methodology Between 2000 and 20104236 children were evaluated retrospectively clinically and by echocardiography for congestive heart failure (CHF) caused by CHD. CHD was diagnosed in 832 patients $(19.6 \%)$ while 78 patients $(1.84 \%)$. Also from the study have been exclude children with complex CHD caused central cyanosis. In all patients were analyzed heart failure symptoms, hemoglobin levels, age of diagnosis, duration of hospitalization per year and death during medical attendance.

Results Anemia was present in $78 \%$ of children with CHD, and in $85 \%$ children with CHF. Hospitalization days per year in anemic patients had a significantly higher than in non-anemic patients (mean $32.5 \pm 39.6$ days per year versus $12.3 \pm 14.3$ days per year $(p<0.05)$. There was not found significant relation onset of heart failure symptoms and the risk of developing anemia between anemic and non-anemic patients. The evaluation showed also needed for the two times longer period of the treatment of patients with CHD with antianemic medication in compare with healthy anemic children.

Conclusion Anemia is prevalent in pediatric patients with CHD especially patients with heart failure. Study showed no higher mortality in anemic patients.

\section{A NOVEL MYH7 VARIANT IDENTIFIED IN A CHILD WITH RESTRICTIVE CARDIOMYOPATHY}

doi:10.1136/archdischild-2012-302724.0510

C Peevers. Paediatric Cardiology, Bristol Children's Hospital, Bristol, UK

Restrictive cardiomyopathy (RCM) is very rare in children and usually associated with a poor prognosis. Identification and thorough 